\title{
EINFLUSS DES LOCKDOWNS AUF PSYCHE UND MEDIENGEBRAUCH IN DER KINDER- UND JUGENDPSYCHIATRIE
}

Die COVID-19-Pandemie stellte alle Familien vor noch nie dagewesene Herausforderungen: Kontaktverbote und das Fehlen von Alternativen der Freizeitgestaltung führten dazu, dass digitale Medien einen grösseren Stellenwert erhielten als je zuvor. Kinder und Jugendliche mit psychischen Vorerkrankungen waren von den Auswirkungen der Pandemie in ganz besonderer Weise betroffen und es bestand die Sorge, es könne gerade in einer Population mit Risiko für Sucht oder dysfunktionalem Verhalten zu einem Anstieg von problematischem Internetgebrauch kommen.

\section{Einfluss des COVID-19 Lockdowns auf die psychische Gesundheit von Kindern und Jugendlichen}

Auch wenn die Infektion mit COVID-19 für Kinder und Jugendliche selbst vergleichsweise ein eher geringeres gesundheitliches Risiko darstellt, wurde die Pandemie besonders für sie nachweislich zu einer grossen psychischen Belastung. Durch umfassende Einschränkungen (u.a. Schliessung von Bildungs-, Betreuungs-, Sport- und Freizeiteinrichtungen, Kontaktbeschränkungen oder -verbote), konnten sie ihrem gewohnten Alltag nicht mehr nachgehen. Dies hatte unmittelbare Auswirkungen auf das psychosoziale und emotionale Befinden von Kindern und Jugendlichen.

Im internationalen Vergleich waren die getroffenen Massnahmen und auch Konsequenzen der Pandemie gerade für Kinder unterschiedlich. Einen kompletten Lockdown mit Aussetzung des Präsenzunterrichts an allen Schultypen gab es - bislang - in der Schweiz lediglich im Frühjahr 2020. Dies war in vielen Ländern in Europa anders, in denen die zweite Welle der Pandemie seit Herbst 2020 mit oft monatelangen Schulschliessungen und strengen Ausgangssperren einherging. In anderen Ländern, vor allem Süd- und Mittelamerika, fand von März 2020 bis März 2021 zum Teil gar kein Schulunterricht mehr statt. Allen gemeinsam war dagegen die Situation der sozialen physischen Isolation in der Freizeit, die verstärkte Konzentration auf die Kernfamilie, oft in räumlicher Enge, sowie Bewegungsmangel aufgrund von Schliessung von Sportund Freizeitmöglichkeiten oder Ausgangssperren.

Viele Studien beschreiben bislang Auswirkungen, welche sich primär auf die Konsequenzen des ersten Lockdowns im Frühjahr 2020 beziehen. Dabei zeigte sich in vielen Studien sowohl eine Zunahme von exter- nalisierenden (Erregbarkeit, Reizbarkeit, Hyperaktivität sowie Aggressivität) als auch internalisierenden Verhaltensauffälligkeiten (emotionaler Stress, Unaufmerksamkeit, Traurigkeit, Schlafstörungen). Vor allem jüngere Kinder machten sich Sorgen, dass Familienangehörige durch COVID-19 infiziert werden könnten, zeigten sich verunsichert und wiesen Symptome von Trennungsangst auf. Auch kämpften Jugendliche während der Pandemie mit Sorgen und Ungewissheit bezüglich ihrer akademischen und persönlichen Zukunft und zeigten vermehrt depressive Symptome.

Allgemein berichteten Kinder und Jugendliche von einer deutlich eingeschränkten Lebensqualität, verstärkter psychischer Belastung und Ängsten. Kinder und Jugendlichen mit niedrigerem sozioökonomischem Status, niedrigerem elterlichen Bildungsniveau sowie Migrationshintergrund waren besonders betroffen, da sie in ihrem familiären Umfeld weniger Unterstützung und Anregung erhielten ${ }^{1)}$. Gerade in Familien mit stärkerer sozialer Belastung, z.B. durch Arbeitsplatzverlust, nahmen Gewalterfahrungen der Kinder zu.

Auswirkung des Lockdowns auf Kinder und Jugendliche mit psychischer Vorerkrankung Die Auswirkungen des Lockdowns und der Pandemie auf Kinder und Jugendliche mit psychischen Vorerkrankungen waren vielfältig. Bei einigen Störungsbildern zeigte sich der Einfluss der Pandemie vermutlich besonders stark: Kinder mit Zwangsstörungen oder hypochondrischen Ängsten waren aufgrund ihrer Symptome wie Kontaminationsangst und Beschäftigung mit dem Körper in der Pandemiesituation vermehrtem Stress ausgesetzt, und gerade jüngere Kinder mit emotionalen Störungen reagierten verstärkt auf die Bedrohung der Familienmitglieder durch Krankheit oder Tod. Depressionen und Ängste wurden durch die Pandemie-Situation verstärkt ${ }^{1}$. Kinder und Jugendliche mit ADHS oder Lernstörungen haben besondere Mühe, selbstorganisiert zu arbeiten oder online Unterricht zu verfolgen; längere Zeiten ohne Präsenzunterricht führen dann zu stärkeren Lernrückständen. Für kleinere Kinder mit besonderem Förderbedarf konnte der Wegfall der spezialisierten Betreuung und die veränderte Tagesroutine zu Rückschritten und verstärkten Verhaltensproblemen führen.

Während die meisten Eltern von Verschlechterungen oder stabilen psychopathologischen Störungs-
Anna Maria Werling

https://doi.org/ $10.35190 / \mathrm{d} 2021.2 .5$
Korrespondenz: anna.werling @pukzh.ch 


\section{Fortbildung}

symptomen während des Lockdowns berichteten, gab es teilweise und insbesondere im ersten Lockdown auch Verbesserungen. Eine kanadische Studie fand zwei bis vier Monate nach Beginn des Lockdowns in etwa 16\% aller Fälle Verbesserung depressiver Symptome bei Jugendlichen mit psychischen Vorerkrankungen, keine Veränderungen bei $52 \%$ und eine Verschlechterung bei 35\% (Elternurteil). Im Selbsturteil fielen die Verbesserungen von depressiven Symptomen mit 20\% leicht höher aus, aber auch die Angabe von Verschlechterungen mit 47\%. Reizbarkeit und Aufmerksamkeitsprobleme wurden ebenfalls häufig von Eltern als verschlechtert beschrieben. Eine französische Befragung während des ersten Lockdowns von Eltern von Patienten mit ADHS kam zu dem Ergebnis, dass etwa ein Drittel der Kinder eine Verbesserung, ein Drittel eine Verschlechterung und ein Drittel keine Veränderung des Verhaltens und Befindens zeigte. Je günstiger die Bedingungen (Garten, ausreichend Platz), desto weniger belastend waren die ADHS Symptome $^{2}$. Jugendliche mit Autismus-Spektrum-Störung zeigten eher eine Verbesserung von Symptomen während des Lockdowns als eine Verschlechterung. Möglicherweise stellten der erzwungene soziale Rückzug und der Wegfall des Präsenzunterrichts für einige Kinder und Jugendliche mit psychischer Vorerkrankung eine deutliche Entlastung dar.

Es ist sicher relevant, zwischen Befunden, die sich auf den Lockdown im Frühjahr 2020 beziehen, und Berichten aus späteren Pandemiephasen zu differenzieren. Stärker noch als während des ersten Lockdowns machte sich auch in der Schweiz während der zweiten Welle der Pandemie ab Herbst 20/Winter 21 ein deutlicher Anstieg der Fallzahlen in Notfallambulanzen der Kinder- und Jugendpsychiatrie bemerkbar. In der Klinik für Kinder- und Jugendpsychiatrie und Psychotherapie der Universität Zürich zeigte sich im letzten Quartal 2020 eine Zunahme um 50\%, die im ersten Quartal 2021 noch weiter anstieg.

In einer britischen Umfrage im Januar/Februar 2021 bei Jugendlichen und jungen Erwachsenen mit psychischen Vorerkrankungen (13 bis 25 Jahre) berichteten $75 \%$, dass sie die aktuelle Situation während des erneuten Lockdowns schwieriger zu bewältigen fanden als beim ersten Lockdown; $44 \%$ fanden es sogar viel schwieriger ${ }^{3)}$. $67 \%$ Prozent rechneten mit längerfristigen Auswirkungen der Pandemie auf ihre psychische Gesundheit, zugleich erwarteten aber 79\% eine Besserung ihrer psychischen Probleme nach der Aufhebung der Einschränkungen.

\section{Mediengebrauch vor und während der Pandemie bei Kindern und Jugendlichen ohne psychische Vorerkrankung}

Die Grenze zwischen adaptiven zu maladaptiven Medienverhalten ist fliessend - nicht nur zu Zeiten einer Pandemie. Frühzeitig den Unterschied zwischen gesunder und problematischer bzw. pathologischer Mediennutzung zu erkennen, stellt Eltern und Fachpersonen vor Herausforderungen. Dabei liegen die Empfehlungen von kinderpsychiatrischen Gremien für den Umgang mit digitalen Medien und die Zahlen der tat- sächlichen Nutzung bei Kindern und Jugendlichen zum Teil deutlich auseinander (siehe Tabelle 1).

Die aktuellsten Zahlen zur durchschnittlichen Nutzung digitaler Medien von Jugendlichen (12-19 Jahre) in der Schweiz wurden online erhoben anhand von Selbstangaben im Mai/Juni 2020, und damit zu einer Zeit zunehmender Lockerungen nach dem Lockdown ${ }^{4}$. Berichtet werden Internetzeiten wochentags von 2 Stunden 44 Minuten, am Wochenende von 3 Stunden 59 Minuten, und Handy-Nutzungszeiten wochentags von 3 Stunden und 47 Minuten, am Wochenende von 5 Stunden und 16 Minuten (jeweils Mittelwerte). Niedriger sozioökonomischer Status und Migrationshintergrund waren mit längeren Medienzeiten assoziiert. Mädchen nutzten das Handy mehr als Knaben; diese spielten dafür häufiger Videospiele als Mädchen (93\% vs. 54\%). Inwiefern die Pandemie die Ergebnisse der Studie beeinflusst hat, die eigentlich auch längsschnittliche Fragen beantworten soll, ist schwer zu sagen; zumindest lässt sich eine gewisse, wenn auch niedrige Zunahme seit der Umfrage von 2018 verzeichnen (2018 wochentags: Internetzeit 2 Stunden 30 Min., Handyzeit 3 Stunden 18 Min.).

Von sehr viel massiveren Zunahmen der Online-Nutzung berichtet dagegen eine Studie aus Deutschland, deren explizites Ziel es war, den veränderten Medien- und Internetgebrauch bei Kindern und Jugendlichen (10-17 Jahre) während des Lockdowns im Frühjahr 2020 im Vergleich zu September 2019 zu erfassen. Demnach kam es bei Kindern und Jugendlichen während des Lockdowns wochentags zu einer Zunahme der Gaming-Zeiten von 75\% und der Social media-Zeit um $66 \%{ }^{9}$. Häufiger als noch vor dem Lockdown wurde als Motivation für Mediennutzung der Wunsch genannt, mit anderen in Kontakt zu bleiben: Mädchen äusserten dies in Bezug auf soziale Medien, Jungen in Bezug auf Gaming (Jungen gamen häufiger mit- oder gegeneinander als Mädchen). Einen besonderen Anstieg im Nutzungsverhalten gab es bei denjenigen, die bereits vorher eine risikoreiche oder pathologische Nutzung gezeigt hatten. Risikoreiches Nutzungsverhalten wurde 2019 bei $10 \%$ der 10 bis 17-jährigen in Bezug auf Gaming und 8.2\% für soziale Medien gefunden, pathologische Nutzung bei $2.5 \%$ für Gaming und 3.2\% für soziale Medien.

Trotzdem ist der Anstieg des Mediengebrauchs während des Lockdowns nicht per se als negativ anzusehen. In kürzlich veröffentlichten Empfehlungen von der WHO wurde dazu aufgerufen, dass Kinder und Jugendliche in Pandemiezeiten über digitale Foren (unter Supervision der Eltern) Kontakt pflegen sollen. Schliesslich wurde nicht nur Schulunterricht, sofern die Voraussetzungen vorhanden waren, digital aufrechterhalten, sondern auch zunehmend Konsultationen über Telepsychiatrie durchgeführt. In vielen Familien wurden digitale Medien als angemessenes Mittel zur Bewältigung der COVID-19 bedingten Einschränkungen eingesetzt, um Kontakte aufrecht zu erhalten, Langeweile zu entgehen und Stress abzubauen. Kinder und Jugendliche haben digitale Medien während des Lockdowns zur Stimmungsregulierung 


\section{Fortbildung}

Kinder- und Jugendpsychiatrische

Medienverhalten gemäss Umfragen (Schweiz)

Empfehlungen für Mediengebrauch ${ }^{5}$

\begin{tabular}{|c|c|}
\hline Vor Schulbeginn nur analog spielen und lernen & $\begin{array}{l}\text { 4- bis 6-jährige: } 29 \% \text { spielen regelmässig } \\
\text { Videogames, } 25 \% \text { nie }^{6)}\end{array}$ \\
\hline $\begin{array}{l}\text { Eigenes Smartphone: nicht vor der } 5 \text {. Klasse }(7 \mathrm{H}) \text {, } \\
\text { danach Nutzung unter elterlicher Steuerung }\end{array}$ & $\begin{array}{l}\text { ca. } 25 \% \text { der 6-9jährigen und ca. } 60 \% \\
\text { der 10-11jährigen haben ein eigenes Handy } 7 \text { ) }\end{array}$ \\
\hline $\begin{array}{l}\text { Maximale Medienzeit pro Tag (insgesamt Handy, } \\
\text { Internet, PC etc.): } \\
\text { - 7-10 Jahre: } 45 \text { Minuten } \\
\text { - 11-13 Jahre: } 1 \text { Stunde } \\
\text { - ab } 14 \text { Jahre: } 1.5 \text { Stunden }\end{array}$ & $\begin{array}{l}\text { Primarschulalter'): im Internet Surfen pro Tag: Nutzer: } \\
16 \text { Minuten, nie: } 57 \% \text { Gamen pro Tag: Nutzer: } \\
24 \text { Minuten, nie: } 43 \% \\
\text { Jugendliches): Handynutzung pro Tag: } 4 \text { Stunden } \\
29 \text { Minuten (Wochenende) }\end{array}$ \\
\hline $\begin{array}{l}\text { PC im eigenen Zimmer: frühestens ab } 12 \text { Jahren } \\
\text { (mit Regeln) }\end{array}$ & $\begin{array}{l}\text { 16\% der 10-/11-Jährigen haben einen PC } \\
\text { im eigenen Zimmer }\end{array}$ \\
\hline $\begin{array}{l}\text { Internetzugang: } \\
\text { ab } 8 \text { Jahren unter Aufsicht } \\
\text { ab } 12 \text { Jahren auch alleine }\end{array}$ & $\begin{array}{l}29 \% \text { der 8-/9-jährigen haben Internetzugang } \\
\text { im eigenen Zimmer?) }\end{array}$ \\
\hline $\begin{array}{l}\text { Chatten: } \\
\text { ab } 8 \text { Jahren unter Aufsicht } \\
\text { ab } 11 \text { Jahren mit Regeln, Kontrolle }\end{array}$ & $\begin{array}{l}\text { Primarschulalter: Messenger Apps: Nutzer } 15 \text { Min. } \\
\text { pro Tag; nie: } 72 \%^{7)} \text { Soziale Medien: Nutzer } 21 \text { Min. } \\
\text { pro Tag; nie: } 91 \%^{7)}\end{array}$ \\
\hline
\end{tabular}

5) Empfehlungen der gemeinsame Suchtkommission der deutschen kinder- und jugendpsychiatrischen Verbände und wissenschaftlichen Fachgesellschaft.

6) ADELE+ Studie 2020 (Elternangaben),

7) MIKE-Studie 2019 (Elternangaben)

8) JAMES-Studie 2018 (Selbstangaben). Das BAG (2021) nennt die 3-6-9-12 Regel als Richtlinie: Kein Fernsehen unter 3 Jahren, keine eigene Spielkonsole vor 6, Internet nach 9 und soziale Netzwerke nach 12.

Tabelle 1. Gegenüberstellung von Kinder- und Jugendpsychiatrischen Empfehlungen zum Mediengebrauch und Medienverhalten in der Schweiz (vor der Pandemie)

(u.a. Ablenkung, Bekämpfung von Langeweile, Bewältigung von Frustration, Flucht aus der Realität), für Informationszwecke oder als Kommunikationsmöglichkeit mit Gleichaltrigen genutzt. Dies führte unweigerlich zu einer gesteigerten Mediennutzung, mit den damit einhergehenden Risiken. Auch von den Eltern wurden digitale Medien als Mittel eingesetzt, Kinder während ihrer eigenen Arbeitszeit im Homeoffice zu beschäftigen.

\section{Definition von problematischem Internetgebrauch}

Problematischer Internet- oder Mediengebrauch, häufig auch «pathologischer Internetgebrauch» (aus dem Englischen «problematic use of the internet, PUI»), wird als Oberbegriff für dysfunktionale Verhaltensweisen verwendet, die im Internet ausgeübt werden bzw. mit dem Gebrauch von digitalen Medien einhergehen, und die zu einer funktionalen Beeinträchtigung im Alltag, etwa im Umgang mit Freunden, Familie, Schule oder Ausbildung führen. Zu den problematischen Verhaltensweisen werden unkontrolliertes Gamen (auch auf Videokonsole, nicht nur online) und die exzessive Nutzung von unterschiedlichsten im Internet verfügbaren Angeboten gezählt, die einen suchtartigen Charakter haben können, wie soziale Medien, Videofilme oder Clips, Online-Shopping, Cyberpornographie. Daneben gibt es problematische oder risikoreiche Verhaltensweisen im Internet wie Cybermobbing, oder Cybergrooming (unerwünschte Kontaktaufnahme mit einem Kind durch Erwachsene mit sexuellen Absichten), Sexting (Versenden freizügiger Bilder der eigenen Person nach Aufforderung) mit potentiell schädlichen Auswirkungen auf die psychische Gesundheit ${ }^{10)}$.

Eine allgemein gültige Definition und Nomenklatur gibt es bisher noch nicht und man findet viele ähnliche Begriffe in der Literatur wie «Mediensucht», «Internetabhängigkeit», «Internetsucht», «Medienabhängigkeit», «Computerabhängigkeit», «Computerspielsucht», «Internetbezogene Störungen». Die Pionierin auf diesem Gebiet, Kimberly Young, hat bereits früh gefordert, dass es sich bei PUI um eine eigenständige Störung im Sinne einer Verhaltenssucht handelt und sie hat die Kriterien für einen pathologischen Internetgebrauch an die DSM-IV-Kriterien des pathologischen Spielens («Glücksspielsucht») angelehnt und ein breites Spektrum von Verhaltensweisen und Impulskontrollproblemen eingeschlossen (exzessives Chatten, Spielen und Handeln im Internet, zwanghafte Informationssuche und Erstellen von Datenbanken, exzessive Cybersexseiten-Nutzung) ${ }^{11)}$.

PUI und substanzgebundene Abhängigkeitsstörungen weisen ebenfalls Gemeinsamkeiten auf, wie Kontrollverlust, Entzugserscheinungen, Toleranzentwicklung, negative Auswirkungen auf soziale Beziehungen, Schule, Arbeit und Leistung. Bislang wurde lediglich die «Internetspielsucht» (Internet Gaming Disorder, IGD) im DSM-5 als klinische Diagnose aufgenommen ${ }^{12}$, deren Kriterien sich an substanzgebun- 


\section{Fortbildung}

denen Abhängigkeitsstörungen orientieren. Im ICD11 ist «Gaming Disorder», als eine Verhaltenssucht mit etwas weniger strengen Kriterien im Vergleich zu IGD, enthalten ${ }^{13)}$. «Hazardous Gaming» ist eine weitere Kategorie im ICD-11, die den Gesundheitsrisiken zugeordnet ist. Die bisherige Beschränkung auf das Gamen bei der Definition von internetbezogenen Störungen ist vielfach kritisiert worden ${ }^{9)}$. Prävalenzangaben für problematisches Internetverhalten, meist auf pathologisches Gamen bezogen, schwanken in epidemiologischen Studien stark: Zwischen $0.21 \%$ in europäischen bis zu $57 \%$ in asiatischen Ländern.

\section{Problematischer Internetgebrauch}

bei Kindern und Jugendlichen mit

psychopathologischen Störungen

Populationsbasierte Studien legen nahe, dass sich ein problematischer Medienkonsum negativ auf die psychische Gesundheit auswirkt. Wenig gut untersucht ist der genaue Zusammenhang von problematischer Internetnutzung und psychischen Störungen im Kindes- und Jugendalter. Ganz generell zeigen Untersuchungen, dass PUI oder PUI-Subtypen häufiger bei Patienten mit psychopathologischen Störungen auftreten als in der allgemeinen Bevölkerung. Auch wenn nicht geklärt ist, ob es eine «reine» Internetabhängigkeit gibt oder sich PUI auf der Basis einer anderen grundlegenden psychiatrischen Störung entwickelt, zeigt PUI eine hohe Komorbidität mit anderen psychiatrischen Störungen ${ }^{10)}$.
Man kann dabei mindestens drei mögliche Mechanismen annehmen:

1. PUI und verschiedene psychopathologische Störungen haben ähnliche Ursachen und teilen Merkmale, sodass das Auftreten der einen Störung mit einem erhöhten Risiko für die andere Störung verknüpft ist. So findet man etwa häufig PUI, vor allem problematisches Gamen, bei Kindern und Jugendlichen mit einer beeinträchtigten Impulskontrolle und Störungen des Belohnungssystems, z. B. im Rahmen von Aufmerksamkeitsdefizit/Hyperaktivitätsstörung (ADHS) oder allgemein bei externalisierenden Störungen. Auch können Zwangsstörungen mit PUI assoziiert sein.

2. Manche PUI-Verhaltensweisen scheinen die Folge oder ein maladaptiver Lösungsversuch eines psychischen Problems zu sein, z. B. die Vermeidung direkter Kontakte bei sozialphobischen oder autistischen PatientInnen. Bei Autismus-Spektrum-Störung und bei Angststörungen geht man daher von erhöhten PUI-Raten aus. Es kann online gelingen Kontakte herzustellen, die im analogen Leben, z.B. aufgrund eingeschränkter sozialer Fertigkeiten, nicht möglich wären. Auch bei niedrigem Selbstwert kann man sich im Netz gut hinter einem virtuellen Ego verstecken.

3. Andere Störungen scheinen sich bei exzessiver/risikoreicher Mediennutzung zu verstärken, wie Ess-

\begin{tabular}{|c|c|c|}
\hline $\begin{array}{l}\text { Internet Gaming Disorder } \\
\text { (Internetspielsucht) }\end{array}$ & $\begin{array}{l}\text { Gaming Disorder } \\
\text { (Videospielabhängigkeit) }\end{array}$ & $\begin{array}{l}\text { Hazardous Gaming } \\
\text { (eigene Übersetzung: riskoreiches } \\
\text { Videospielen) }\end{array}$ \\
\hline DSM-5 ${ }^{12)}$ & ICD-1113 & ICD-11 $^{13)}$ \\
\hline $\begin{array}{l}\text { - Gedankliche Eingenommenheit } \\
\text { - Entzugssymptomatik bei } \\
\text { Konsumverhinderung } \\
\text { - Toleranzentwicklung } \\
\text { - Kontrollverlust, erfolglose } \\
\text { Abstinenzversuche } \\
\text { - Computerspielnutzung als } \\
\text { dominierende Beschäftigung } \\
\text { und Interessensverlust bei } \\
\text { Hobbys und anderen Aktivitäten } \\
\text { - Fortführung der exzessiven } \\
\text { Nutzung trotz negativer psycho- } \\
\text { sozialer Konsequenzen } \\
\text { - Verheimlichung des Nutzungs- } \\
\text { ausmasses } \\
\text { - Spielen als Emotionsregulation } \\
\text { - Gefährdung zwischenmenschli- } \\
\text { cher Beziehung oder Lebens- } \\
\text { bereiche } \\
\text { Für die Diagnose müssen } 5 \text { oder } \\
\text { mehr der } 9 \text { Kriterien erfüllt sein, } \\
\text { mind. } 12 \text { Monate lang. }\end{array}$ & $\begin{array}{l}\text { - Kontrollverlust über die Verhal- } \\
\text { tensausführung } \\
\text { - Priorisierung der Videospiele, d.h. } \\
\text { andere Lebensbereiche, Inter- } \\
\text { essensfelder und Alltagsaktivitä- } \\
\text { ten werden vernachlässigt oder } \\
\text { verdrängt } \\
\text { - Fortführung der Nutzung von } \\
\text { Videospielen trotz negativer Kon- } \\
\text { sequenzen } \\
\text { - Zusätzlich: Verhalten führt zu } \\
\text { einer schweren Beeinträchtigung } \\
\text { des psychosozialen Funktionsni- } \\
\text { veaus (Familie, Ausbildung, Beruf, } \\
\text { Freizeit) }\end{array}$ & $\begin{array}{l}\text { - Videospielverhalten (online oder } \\
\text { offline) erhöht eigene Gesund- } \\
\text { heitsrisiken } \\
\text { - Beeinträchtigung des Individu- } \\
\text { ums oder Personen aus dem } \\
\text { Umfeld } \\
\text { - Erhöhtes Risiko aufgrund von: } \\
\text { - Häufigkeit und Zeitumfangs von } \\
\text { Gaming } \\
\text { - Vernachlässigung anderer } \\
\text { Aktivitäten, riskantem Verhalten } \\
\text { im Zusammenhang mit Gaming } \\
\text { - negativen Konsequenzen } \\
\text { - Fortführung des Spielens trotz } \\
\text { bekannter Risiken }\end{array}$ \\
\hline
\end{tabular}

Tabelle 2. Gegenüberstellung der Diagnosekriterien von «Internet Gaming Disorder» (Internetspielsucht), «Gaming Disorder» (Videospielabhängigkeit) und «Hazardous Gaming» 


\section{Fortbildung}

störungen oder Depression; andere entstehen möglicherweise sogar neu, z. B. Probleme in der Folge von Schlafmangel, Aufmerksamkeitsprobleme, Leistungsprobleme, Lernprobleme, Adipositas, Kopfschmerzen. Cybermobbing trifft besonders oft Aussenseiter oder Angehörige von sexuellen oder anderen Minderheiten und kann das Entstehen psychischer Störungen begünstigen, bis hin zu Suizidalität.

\section{Nutzung digitaler Medien bei Kindern und Jugendlichen mit psychischer Erkrankung während des Lockdowns - Umfrage bei Patienten der KJPP Zürich}

Um zu untersuchen, wie sich in der Schweiz der Lockdown auf das Medienverhalten und Befinden von Kindern und Jugendlichen mit psychopathologischen Störungen auswirkt, wurde im Frühsommer 2020 an der KJPP Zürich eine Online-Befragung von Patienten und deren Eltern durchgeführt ${ }^{14)}$. Dabei wurden Patienten (Alter 10-18 Jahre) angefragt, die im Laufe der letzten zwei Jahre in einem der acht Ambulatorien der Klinik für Kinder- und Jugendpsychiatrie und Psychotherapie, Universität Zürich, oder in der Spezialambulanz für Autismus eine Behandlung begonnen hatten. Die Befragung fand anonym statt und die Patienten bzw. deren Eltern wurden per Email zur Teilnahme eingeladen. Es handelt sich dabei um eine Stichprobe, die keinen Anspruch auf Repräsentativität erhebt. Aufgrund der Rekrutierungswege (Voraussetzung von Internetzugang sowie Deutsch als Fragebogensprache) und der demographischen Daten wird davon ausgegangen, dass Familien mit niedrigem sozioökonomischem Status unterrepräsentiert sind. Die Erhebung fand überwiegend im Juni 2020 statt und fiel somit in die Zeit, als noch Einschränkungen in Kraft, aber die Schulen meist wieder geöffnet waren.

Die Befragung hatte zwei Schwerpunkte:

1. Fragen zu Medienkonsum und problematischem Internetverhalten. Hierbei stützen wir uns auf ein Verfahren aus Fragen zu problematischem Medienund Internetverhalten, das an unserer Klinik zum routinemässigen Screening in der Kinder- und Jugendpsychiatrie entwickelt wurde.

2. Fragen zur allgemeinen Befindlichkeit, emotionalen und Verhaltenssymptomen während des Lockdowns. Dazu wurde im Rahmen eines europäischen Kooperationsprojekts zusätzlich auf einen Fragebogen zurückgegriffen (CRISIS) (Coghill et al. unveröffentlicht). Da die Umfrage vollständig anonym durchgeführt wurde, beruhten alle demographischen und Störungsangaben auf den Antworten der Eltern.

Von den 477 Elternantworten bezogen sich etwa ein Drittel auf Patienten mit internalisierenden Störungen (Depression und Ängste), ein Drittel auf Patienten mit externalisierenden Störungen (ADHS und Aggressivität) und ein Drittel auf sonstige Störungen (Autismus-Spektrum-Störung, Psychose, Genderdysphorie, Essstörungen, Zwänge und anderes). Exzessi- ves Gaming wurde bei nur drei Patienten als Zuweisungsgrund an die KJPP genannt. Was den Einfluss des Lockdowns auf die Störung anging, gab die Mehrheit (41\%) der Eltern an, dass es während des Lockdowns keine Veränderung des psychopathologischen Hauptproblems gegeben habe, $21 \%$ gaben eine Verschlechterung an und 38\% eine Verbesserung. Unter den Patienten mit internalisierenden Störungen war die Anzahl der Verbesserungen am höchsten (44\%). Dies deckt sich mit anderen Studien, die bei einem Teil der Patienten mit Ängsten und sozialen Problemen von einer Entlastung während des Lockdowns berichten.

Beim Medienkonsum fand sich während des Lockdowns eine Zunahme der täglichen Medienzeit Handy, PC, Tablet, Videokonsole und Fernsehen - um 59 Prozent. Bei Jungen betraf dies vor allem die mit Gamen verbrachte Zeit, bei Mädchen die Zeit in den sozialen Medien. Generell war die Medienzeit bei Jugendlichen sehr viel höher als bei Kindern. Nur bei den Kindern, nicht aber bei den Jugendlichen, gab es einen Zusammenhang zwischen Medienzeit und Verschlechterung der jeweiligen Störung. Betrachtete man dagegen nicht die Gesamtgruppe, sondern nur die Kinder und Jugendlichen mit ADHS, zeigte sich bei beiden ein Zusammenhang zwischen Medienzeit, verminderter Konzentrationsfähigkeit und erhöhter Reizbarkeit ${ }^{15)}$.

Eltern berichteten auch über eine leicht erhöhte Besorgnis während des Lockdowns hinsichtlich suchtähnlicher Verhaltensweisen, wie aggressive und wütende Reaktionen, wenn die Medienzeit durch die Eltern eingeschränkt wurde. Das weist auf die gesteigerte Bedeutung hin, die digitale Medien für die Kinder und Jugendlichen während dieser Phase hatten, möglicherweise aber auch auf die stärkere Aufmerksamkeit der Eltern auf den Medienkonsum ihrer Kinder. Praktisch keine Veränderungen während des Lockdowns gaben die Eltern an bei digitalem Problem- und Risikoverhalten, wie dem sorglosen Umgang mit eigenen Daten oder Cybermobbing. Die Eltern wurden auch befragt, ob sie während des Lockdowns Medienregeln - sofern in der Familie vorhanden - geändert und ihrem Kind mehr Zugang zu Medien erlaubt hätten. Dies wurde von einer deutlichen Mehrheit bejaht und nur von $18 \%$ verneint. Etwa weitere $20 \%$ der Befragten gaben an, dass ihr Kind über die Mediennutzung selbst entscheiden würde.

\section{Zusammenfassung und Ausblick}

Für Kinder und Jugendliche stellte die COVID-19 Pandemie und der Lockdown im Frühjahr 2020 eine Zeit besonderer psychischer Belastung dar, die mit einer Erhöhung psychischer Störungen einherzugehen scheint. Dies trifft im Prinzip noch stärker auf Kinder und Jugendliche mit psychischen Vorerkrankungen zu. Allerdings findet sich hier auch eine Untergruppe, für die der erzwungene soziale Rückzug mit Entlastung und Stressreduktion einhergeht.

Die meisten Untersuchungen zeigen generell einen massiv erhöhten Medienkonsum während des 
Lockdowns unter Kindern und Jugendlichen mit und ohne psychische Vorerkrankungen. Dabei ist davon auszugehen, dass ein Teil dieses Medienkonsums in der Pandemie-Situation durchaus situativ funktional war und dass es nun vor allem bei den bereits vorher gefährdeten Kindern und Jugendlichen zu einem pathologischen Gebrauch kam. Inwieweit Kinder und Jugendliche durch die Pandemie auch nachhaltig beeinträchtigt wurden - was psychopathologische Störungen und das Medienverhalten angeht -, lässt sich derzeit noch nicht abschliessend klären und muss durch Längsschnittstudien genauer untersucht werden.

\section{Danksagung}

Die Autorinnen bedanken sich für die Unterstützung und Zusammenarbeit des Europäischen Netzwerks für Problematische Nutzung des Internets der COST-Aktion CA16207 (Europäische Zusammenarbeit in Wissenschaft und Technologie).

\section{Referenzen}

1) Ravens-Sieberer U, Kaman A, Erhart M, Devine J, Schlack R, and Otto C. Impact of the COVID-19 pandemic on quality of life and mental health in children and adolescents in Germany. European child \& adolescent psychiatry.2021;1-11.

2) Bobo E, Lin L, Acquaviva E, Caci H, Franc N, Gamon L, et al. How do children and adolescents with Attention Deficit Hyperactivity Disorder (ADHD) experience lockdown during the COVID-19 outbreak? Líencephale. 2020;46(3S):S85-S92.

3) YoungMinds. Coronavirus: Impact on young people with mental health needs. 2021

4) Bernath J, Suter L, Waller G, Külling C, Willemse I, and Süss D. JAMES: Jugend, Aktivitäten, Medien-Erhebung Schweiz. 2020.
5) Paschke K, Thomasius R, Holtmann M, Klein M, Melchers P, Schimansky $\mathrm{G}$ et al. Medienkonsum bei Kindern und Jugendlichen-Positionspapier der Gemeinsamen Suchtkommission der kinder-und jugendpsychiatrischen Fachgesellschaft und Verbände (DGKJP, BAG KJPP, BKJPP), Suchttherapie 2019;20: S14-02.

6) Bernath J, Waller G, Meider U. ADELE+: der Medienumgang von Kindern im Vorschulalter (4-6 Jahre)-Chancen und Risiken für die Gesundheit. 2020.

7) Süss D, Waller G, Suter L, Bernath J, Külling C, Willemse et al. Ergebnisbericht zur MIKE-Studie 2019. MIKE. 2019.

8) Suter L, Waller G, Bernath J, Külling C, Willemse I, and Süss D. JAMES: Jugend, Aktivitäten, Medien-Erhebung Schweiz. 2018.

9) DAK-Studie. DAK-Studie: Gaming, Social-Media \& Corona. https://www.dak.de/dak/gesundheit/dak-studie-gaming-socialmedia-und-corona-2295548.html. 2020.

10) Fineberg NA, Demetrovics Z, Stein DJ, loannidis K, Potenza MN, Grunblatt E, et al. Manifesto for a European research network into Problematic Usage of the Internet. Eur Neuropsychopharmacol. 2018;28:1232-46.

11) Young KS. Internet addiction: The emergence of a new clinical disorder. Cyberpsychology \& behavior. 1998;1:237-44.

12) APA (American Psychological Association). Diagnostic and statistical manual of mental disorders (DSM-5). 2013.

13) WHO (World Health Organization). 6C51 Gaming disorder. ICD-11 Beta Draft. 2018. https://icd.who.int/dev11/I-m/en\#/http://id. who.int/icd/entity/1448597234 (Retrieved April 7, 2018).

14) Werling AM, Walitza $S$, Grünblatt $E$, Drechsler R. eingereicht. Media use before, during and after COVID-19 lockdown in a clinically referred sample in child and adolescent psychiatry: Results of an online survey in Switzerland.

15) Werling AM, Walitza S, Drechsler R. Impact of the COVID-19 lockdown on screen media use in patients referred for ADHD to child and adolescent psychiatry. An introduction to problematic use of the internet in ADHD and results of a survey. Journal of Neutransmission. 2021. DOI 10.1007/s00702-021-02332-0

\section{Autorinnen}

Dr. med. Anna Maria Werling, Klinik für Kinder- und Jugendpsychiatrie und Psychotherapie, Psychiatrische Universitätsklinik Zürich, Universität Zürich

Prof. Dr. med. Susanne Walitza, Klinik für Kinder- und Jugendpsychiatrie und Psychotherapie, Psychiatrische Universitätsklinik Zürich, Universität Zürich \& Zentrum für Neurowissenschaften Zürich, Universität und ETH Zürich

Prof. Dr. phil. Renate Drechsler, Klinik für Kinder- und Jugendpsychiatrie und Psychotherapie, Psychiatrische Universitätsklinik Zürich, Universität Zürich

Die Autorinnen haben keine finanziellen oder persönlichen Verbindungen im Zusammenhang mit diesem Beitrag deklariert.

\section{Weltstillwoche 2021:}

\section{MATERIAL FÜR AKTIVITÄTEN}

Planen Sie in Ihrem Verband, Unternehmen oder in Ihrem persönlichen Umfeld eine Veranstaltung zur Weltstillwoche?

Stillförderung Schweiz stellt kostenlos Material zur Verfügung, bestellbar ab sofort:

\section{www.stillfoerderung.ch}

